\title{
Limited Systemic Exposure with Topical Glycopyrronium Tosylate in Primary Axillary Hyperhidrosis
}

\author{
David M. Pariser ${ }^{1} \cdot$ Edward L. Lain $^{2} \cdot$ Richard D. Mamelok ${ }^{3} \cdot$ Janice Drew $^{4} \cdot$ Diane R. Mould $^{5}$
}

Accepted: 5 December 2020 / Published online: 12 January 2021

(c) The Author(s) 2021

\begin{abstract}
Background Glycopyrronium tosylate (GT; Qbrexza ${ }^{\circledR}$ [glycopyrronium] cloth, 2.4\%) is a topical anticholinergic approved (USA) for primary axillary hyperhidrosis in patients aged $\geq 9$ years.

Objective The objective of this study was to compare the pharmacokinetics and safety of GT to oral glycopyrrolate (phase I study) and assess the relationship between glycopyrronium pharmacokinetics and anticholinergic-related adverse events or efficacy with population pharmacokinetics using data from two phase II studies.

Methods In the phase I study, study staff applied GT to axillae of patients with primary axillary hyperhidrosis (aged 9-65 years) once daily (5 days); oral glycopyrrolate was administered to healthy adults (aged 18-65 years) every 8 hours (15 days). In the phase II studies (NCT02016885 [20 December, 2013], NCT02129660 [2 May, 2014]), adults with primary axillary hyperhidrosis applied topical glycopyrronium (0.8-3.2\%) or vehicle to axillae once daily (4 weeks). Pharmacokinetic and adverse event data were collected in all studies.

Results Glycopyrronium pharmacokinetic parameters were similar between adult and pediatric patients treated with GT; there was no evidence of accumulation. Systemic absorption of glycopyrronium was lower with GT vs oral glycopyrrolate. No anticholinergic-related adverse events occurred with GT in the phase I study, while dry mouth and nasal dryness occurred with oral glycopyrrolate; anticholinergic adverse events occurred in the phase II studies. In the population pharmacokinetic analysis, frequency/severity of anticholinergic-related adverse events increased with higher glycopyrronium concentration; no relationship was observed between efficacy and pharmacokinetic measures.

Conclusions These studies indicate limited absorption of GT compared to oral glycopyrrolate and a low risk of anticholinergic adverse events with proper GT administration when following instructions for use (wipe each underarm once with same cloth, wash hands, avoid ocular contact).
\end{abstract}

\section{Introduction}

Supplementary Information The online version contains supplementary material available at https://doi.org/10.1007/s4026 2-020-00975-y.

David M. Pariser

dpariser@pariserderm.com

1 Eastern Virginia Medical School and Virginia Clinical Research, Inc., 6160 Kempsville Circle, Suite 200A, Norfolk, VA 23502, USA

2 Austin Institute for Clinical Research, Austin, TX, USA

3 Mamelok Consulting, Palo Alto, CA, USA

4 Dermira, Inc., a wholly owned subsidiary of Eli Lilly and Company, Menlo Park, CA, USA

5 Projections Research, Inc., Phoenixville, PA, USA
Hyperhidrosis is sweat production in excess of what is required for thermoregulation and is thought to result from overstimulation of eccrine sweat glands by cholinergic sympathetic innervation [1-3]. Hyperhidrosis affects an estimated $4.8 \%$ of the US population (approximately 15.3 million people) with accompanying negative impacts to quality of life [3-7]. A number of treatments have been tried for hyperhidrosis, including oral anticholinergics [5, 8]. Oral anticholinergics have not been approved for hyperhidrosis, and their clinical utility is often limited by anticholinergic adverse events (AEs) at doses often required for efficacy [5]. The potential for reduced systemic exposure from a locally acting, topical anticholinergic may allow for a more favorable safety profile. 


\section{Key Points}

Glycopyrronium tosylate (GT) $2.4 \%$ cloth is a oncedaily topical anticholinergic available in the USA for the treatment of primary axillary hyperhidrosis in adult and pediatric patients 9 years of age and older.

In a phase I study, systemic absorption of glycopyrronium and the rate of adverse events were lower with GT vs oral glycopyrrolate; in a population pharmacokinetic analysis using data from two phase II studies, the probability of having an anticholinergic adverse event with GT was related to plasma concentrations of glycopyrronium, which were generally low.

Overall in these studies, there was limited absorption and a corresponding low risk of anticholinergic adverse events with proper GT administration, which is consistent with phase III clinical observations and highlights the importance of GT instructions for use, which include wiping each underarm once with the same cloth, washing hands with soap and water immediately after discarding the used cloth, and avoiding contact with the eyes.

Glycopyrronium tosylate (GT) is a once-daily topical anticholinergic approved in the USA (2018) for the treatment of primary axillary hyperhidrosis in patients aged $\geq 9$ years $\left(\right.$ Qbrexza $^{\circledR}$ [glycopyrronium] cloth, $2.4 \%$ ) [9-13]. In two phase III, randomized, double-blind, vehicle-controlled, replicate 4-week trials (NCT02530281 and NCT02530294), GT significantly reduced sweating severity and sweat production compared with vehicle and improved dermatology-related quality-of-life measures. The most common AEs ( $\geq 5 \%$ ) were dry mouth, application-site pain, mydriasis, oropharyngeal pain, and headache $[10,12,14]$. Patients are to apply GT once daily by using a single cloth to wipe across each of their axilla one time. In a treatment algorithm for primary axillary hyperhidrosis developed by the International Hyperhidrosis Society, GT and topical antiperspirants are recommended as first-line treatment [15].

While topical administration can reduce overall drug exposure, characterization of the relationship of the pharmacokinetic (PK) profile to efficacy and safety will allow for a better understanding of clinical effects. The objectives of the present studies are (1) to compare the pharmacokinetics, safety, and tolerability of topical GT and oral glycopyrrolate solution in an open-label, phase I study and (2) to evaluate the relationship of the topical glycopyrronium PK profile to anticholinergic-related AEs and efficacy using a population PK (Pop PK) and pharmacodynamic model applied to data from two phase II studies (HH01 [NCT02016885], HH02 [NCT02129660]).

\section{Methods}

For the phase I and II clinical studies described here, the protocols, informed consent forms, and assent forms for subjects under legal adult age (phase I study only) were submitted to an institutional review board or independent ethics committee for review and approval before the start of the study. The studies were conducted in compliance with the principles of the International Conference on Harmonisation (E6 1997) Good Clinical Practice, US Food and Drug Administration guidelines, and the Declaration of Helsinki, 1964 (as amended in Edinburgh [2000]), and informed consent was obtained.

\subsection{Phase I PK Study}

\subsubsection{Study Design and Subjects}

Seven study centers in the USA screened and enrolled subjects (six sites enrolled subjects in the GT arm and one site enrolled subjects in the oral glycopyrrolate arm). The first subject enrolled on 2 May, 2016, and the last subject completed on 20 July, 2016.

In the phase I, open-label study, GT $2.4 \%$ cloth was applied topically once daily for 5 days to both axillae of patients aged 9-65 years with primary axillary hyperhidrosis, while three doses of oral glycopyrrolate solution $\left(\right.$ Cuvposa $\left.^{\circledR}\right)$ were administered to healthy adult volunteers aged 18-65 years every $8 \mathrm{~h}$ for 5 days for each dose (Fig. 1). GT was applied by study staff to control for application method and prevent non-axillary exposure, and GT was administered by wiping each axilla once using the same GT cloth. Oral glycopyrrolate was administered based on the prescribing information for Cuvposa ${ }^{\circledR}$ [16] starting at $1 \mathrm{mg} / 8 \mathrm{~h}$ on day 1 and titrated in $1-\mathrm{mg}$ increments every 5 days to a maximum dose of $3 \mathrm{mg} / 8 \mathrm{~h}$ if no dose-limiting side effects occurred. Subjects in the oral glycopyrrolate arm were required to fast for $\geq 8 \mathrm{~h}$ prior to the first daily dose, $\geq 2 \mathrm{~h}$ prior to the second and third daily doses, and $\geq 3 \mathrm{~h}$ following each dose; water was withheld for $\geq 2 \mathrm{~h}$ prior to and following all doses. Subjects in the GT arm returned to the study center on each treatment day (days 1-5) and on day 6 (study exit); a safety telephone followup call visit was conducted on day 7 or 2 days after early termination. Subjects in the oral glycopyrrolate arm were housed in a study unit from day - 1 through day 16 (study exit) or early termination; a safety follow-up telephone call was conducted on day 17 or 2 days after the subject stopped oral glycopyrrolate dosing. 


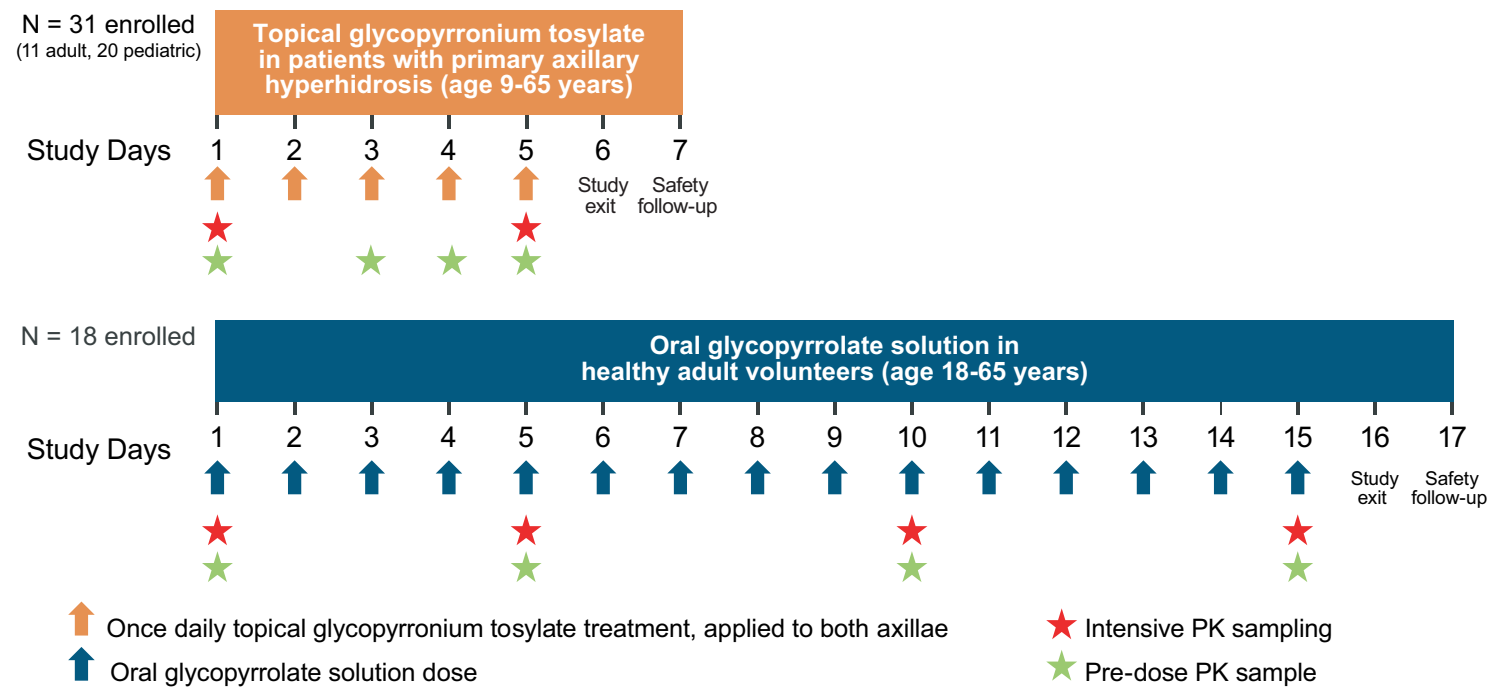

Fig. 1 Phase I study design. Study staff administered all the study drug. Topical glycopyrronium tosylate (GT; $2.4 \%$ ) was applied to each axilla once daily. Because of the oral glycopyrrolate titration schedule $(1.0 \mathrm{mg} / 8 \mathrm{~h}$, then increased by $1.0-\mathrm{mg}$ increments every 5 days to a maximum of $3.0 \mathrm{mg} / 8 \mathrm{~h}$ if no dose-limiting side effects), the oral glycopyrrolate arm was 17 days long whereas the GT arm, which did not involve titration, was 7 days long. Pediatric patients (included

Patients eligible for the GT arm were male or non-pregnant, non-lactating female patients with primary axillary hyperhidrosis for $\geq 6$ months, gravimetrically measured sweat production of $\geq 50 \mathrm{mg} / 5 \mathrm{~min}$ in each axilla, Axillary Sweating Daily Diary [17] Item 2 (severity) score $\geq 4$ (0-10 numeric rating scale), and Hyperhidrosis Disease Severity Scale (HDSS) grade 3 or 4. Pediatric patients (ages 9 to $<18$ years) who were participating in a phase III, openlabel extension trial of GT (NCT02553798) were allowed to participate concurrently in this phase I study; these patients underwent a 7-day wash-out of GT prior to day 1 of this study. The study drug from the phase III, open-label extension trial of GT was withheld throughout the subject's participation in the phase I study and resumed following the day 6 study exit visit. Patient exclusion criteria for the GT arm included prior surgical procedure for hyperhidrosis, prior axillary treatment with an anti-hyperhidrosis medical device, prior treatment with axillary iontophoresis within 4 weeks of day 1 or botulinum toxin (e.g., Botox ${ }^{\circledR}$ ) for axillary hyperhidrosis within 1 year of day 1 , axillary use of nonprescription antiperspirants within 1 week or prescription antiperspirants within 2 weeks of day 1 , secondary axillary hyperhidrosis or presence of a condition that may cause secondary hyperhidrosis (e.g., lymphoma, malaria, severe anxiety not controlled by medication, carcinoid syndrome, substance abuse, hyperthyroidism), or history of Sjögren's syndrome, Sicca syndrome, or glaucoma. only in the GT arm; age 9 to $<18$ years) had a modified pharmacokinetic (PK) sampling schedule to comply with guidelines for safe volumes of blood sampling; no pre-dose samples were collected in pediatric subjects on days 2,3 , and 4 . In case of early termination, the safety follow-up telephone call occurred 2 days after early termination

Patients eligible for the oral glycopyrrolate arm were healthy male or non-pregnant, non-lactating female patients as assessed by history, physical exam, vital signs, electrocardiography, and clinical laboratory tests at screening and day -1 , with weight of $\geq 48 \mathrm{~kg}$ and a body mass index $\geq 18.0$ $\mathrm{kg} / \mathrm{m}^{2}$ and $\leq 33.0 \mathrm{~kg} / \mathrm{m}^{2}$ at screening and day -1 . Exclusion criteria included history or manifestations of clinically significant urological, gastrointestinal, renal, hepatic, neurological, hematological, metabolic, oncologic, pulmonary, immunologic, psychiatric, or cardiovascular disease or disorder, history of any medical or surgical conditions with potential to interfere with or alter the gastrointestinal absorption, distribution, metabolism, or excretion of the study drug, history of Sjögren's syndrome or Sicca syndrome, history of glaucoma, inflammatory bowel disease, toxic megacolon, or recent febrile illness (within 14 days and $>100.0{ }^{\circ} \mathrm{F}$ ), male patients with a history of urinary retention requiring catheterization because of prostatic hypertrophy or severe obstructive symptoms of prostatic hypertrophy, evidence of urinary obstruction or difficulty in voiding at screening, and taking any drug containing glycopyrrolate within 4 weeks of study entry.

\subsubsection{Blood Sample Collection and Analysis}

For all samples, approximately $3 \mathrm{~mL}$ and $2 \mathrm{~mL}$ of blood was collected from adults and pediatric patients, respectively. In 
patients treated with GT, blood samples were collected on days 1-5 (Fig. 1). Differences in sample collection between adult and pediatric patients because of guidelines regarding safe volumes of blood sampling in pediatric patients ( 9 to $<18$ years of age) are noted, where applicable, below. All GT-treated patients underwent intensive PK sampling on days 1 and 5: samples were collected prior to the application of the study drug (pre-dose) and $30 \mathrm{~min}, 1,1.5,2,2.5$, 3,4 , and $6 \mathrm{~h}$ post-application; additional samples were collected in adults at 8,10 , and $24 \mathrm{~h}$ post-application. Pre-dose samples were collected from adults on days 3 and 4 , and no samples were collected from pediatric patients on days 2, 3, and 4. Oral glycopyrrolate-treated subjects underwent intensive PK sampling on days 1, 5, 10, and 15 (Fig. 1). On these days, blood samples were collected pre-dose and 30 min, 1, 1.5, 2, 2.5, 3, 4, 6, 8, 10, and $24 \mathrm{~h}$ post-dose.

Plasma concentrations of glycopyrronium were determined using a validated liquid chromatography-tandem mass spectrometry method. Assays were performed by PPD Laboratories (Richmond, VA, USA). The validated lower limit of quantification (LLOQ) of the plasma assay for glycopyrronium was $0.01 \mathrm{ng} / \mathrm{mL}$. The PK evaluable population included subjects who received the study drug and had one or more PK samples collected. As specified in the statistical analysis plan, samples were excluded from the analysis if there were plasma concentration values $>3$ standard deviations from the mean value for a given timepoint. Pre-dose samples on day 1 were set to 0 . Noncompartmental PK analysis was conducted using WinNonlin (version 6.4, Pharsight Corp., Mountain View, CA, USA). The maximum plasma concentration $\left(C_{\max }\right)$, time to $C_{\max }\left(T_{\max }\right)$, area under the plasma concentration vs time curve (AUC) from 0 to $24 \mathrm{~h}$ $\left(\mathrm{AUC}_{0-24 \mathrm{~h}}\right.$ ), AUC from 0 to $6 \mathrm{~h}$, and elimination (terminal) half-life were determined.

Comparisons of exposure metrics $\left(C_{\max }, \mathrm{AUC}\right)$ for topical GT vs oral glycopyrrolate were evaluated using the ln-transformed parameters of test (GT) to reference (oral glycopyrrolate). Data from the two treatments were analyzed using an analysis of variance model with a fixed-effect term for formulation (topical vs oral) using $\mathrm{R}$ (programming language, version 3.2.0 [https://www.r-project.org/]). Least-squares mean differences and their $90 \%$ confidence intervals (CIs) in the logarithmic scale were back transformed to produce geometric mean ratios (point estimates) and associated $90 \%$ confidence intervals (CIs) for each ratio. A similar comparison of exposure metrics for pediatric patients treated with GT (test) and adult patients treated with GT (reference) was performed.

\subsubsection{Safety}

Safety assessments included treatment-emergent adverse events (TEAEs) and local skin reactions (GT arm only).
Adverse events were collected after the first dose of study drug was applied. Subjects were instructed to inform the investigator and/or study staff of any AE. At each visit, subjects were asked about AEs in a nonspecific manner using open-ended questions so as not to bias the response (e.g., "How have you been since the last visit?"). Adverse events were also assessed during the safety telephone follow-up call visit (day 7 for GT and day 17 for oral glycopyrrolate, or 2 days after early termination). Anticholinergic AEs were identified by medical review of reported events without knowledge of the patients in which these events occurred. The safety population included all enrolled subjects who received one or more confirmed dose of the study drug. Local skin reactions included burning/stinging, pruritus, edema, erythema, dryness, and scaling and were assessed pre-dose on day 1, post-dose following each GT application, and day 6 .

\subsection{Population PK Study}

\subsubsection{Study Design and Subjects}

The Pop PK analysis was performed with data from two randomized, double-blind, vehicle-controlled, phase II studies conducted in adults (aged $\geq 18$ years) with primary axillary hyperhidrosis (DRM04-HH01 [NCT02016885] and DRM04-HH02 [NCT02129660]); Fig. 1 of the Electronic Supplementary Material [ESM]). Patients were randomized at 20 study centers in the USA for DRM04-HH01 (first subject enrolled 10 December, 2013; last subject completed 15 July, 2014) and at 15 study centers in the USA and Canada for DRM04-HH02 (first subject enrolled 9 April, 2014; last subject completed 5 November, 2014). In both trials, eligible patients had primary axillary hyperhidrosis for $\geq 6$ months, gravimetrically measured sweat production of $\geq 50 \mathrm{mg} / 5 \mathrm{~min}$ in each axilla, and HDSS grade 3 or 4 . Results from a prior proof-of-concept study ( $n$ $=38$ ) with glycopyrronium bromide $2 \%$ and $4 \%$ informed the doses selected for study in the two phase II trials. In DRM04-HH01, patients were randomized 1:1:1:1:1 to one of four doses of topical glycopyrronium bromide $(0.8 \%$, $1.6 \%, 2.4 \%, 3.2 \%$ ) or vehicle. Patients in DRM04-HH02 were randomized 1:1:1:1:1 to one of two doses of GT $(1.6 \%, 2.4 \%)$, one of two doses of glycopyrronium bromide $(1.6 \%, 2.4 \%)$, or vehicle. Both glycopyrronium bromide and GT contain the glycopyrronium cation, which is the active anticholinergic moiety $[18,19]$. In both trials, patients were to apply the study drug to the axillae once daily in the evening (wiping each axilla once using the same cloth) for 4 weeks with a 2-week off-drug follow-up. Subjects returned to the study center at weeks 1, 2, 3, 4 (end of treatment), 5, and 6 (study exit). 


\subsubsection{Blood Sample Collection and Analysis}

For all samples, at least $500 \mu \mathrm{L}$ of plasma (at least $1 \mathrm{~mL}$ of blood) was collected. For DRM04-HH01, blood samples were collected pre-dose and at approximately $30 \mathrm{~min}, 1$, $2,3,4$, and $24 \mathrm{~h}$ after application on day 1 . Trough blood samples were collected pre-dose at the end of weeks 1, 2, 3, and 4. For DRM04-HH02, approximately 10 subjects were to provide PK samples at the baseline/day 1 visit (pre-dose and $30 \mathrm{~min}, 1,1.5,2,2.5,3,3.5,4,5,6,8,10,12$, and 24 $\mathrm{h}$ post-dose) and pre-dose samples at weeks 4,5 , and 6 . Approximately 20 subjects were to provide PK samples at the baseline/day 1 visit (pre-dose samples), at the week 2 visit (pre-dose and $30 \mathrm{~min}, 1,1.5,2,2.5,3,3.5,4,5,6,8$, 10,12 , and $24 \mathrm{~h}$ post-dose), and pre-dose samples at weeks 4,5 , and 6. All other patients in DRM04-HH02 were to provide pre-dose blood samples on day 1 and at the weekly visits through week 6. Plasma concentrations of glycopyrronium were determined using a sensitive and selective liquid chromatography-tandem mass spectrometry method. Assays were performed by PPD Laboratories. The validated LLOQ of the plasma assay for glycopyrronium was $0.02 \mathrm{ng} /$ $\mathrm{mL}$. The PK population was defined as subjects who had blood collected for a PK analysis pre-dose and at least once post-dose.

\subsubsection{Population PK Model Development}

All glycopyrronium concentration data from the active treatment arms of DRM04-HH01 and DRM04-HH02 were pooled into a single database (data from vehicle arms were not included in the Pop PK model development). Pre-dose samples were excluded from the database as were samples for which no results were reported, observations were missing, or the conditional weighted residual was $>5$ and the key parameter estimates differed by more than $15 \%$ or impacted on the convergence of the models. For missing data, imputation strategies were implemented; for example, if the covariate value was missing at a given time/visit, it was imputed using the nearest assessment. The database was used to create a Pop PK model for glycopyrronium administered topically using NONMEM (Version 7.2.0, Icon PLC, Dublin, Ireland). The Pop PK model was developed in steps; a base model for description of the structural components of the model, a full model including all of the pre-specified covariate effects of interest, then the final model chosen by retaining only the statistically significant covariate effects. A one-compartment model with first-order input and elimination was used as the base Pop PK model. Given the number of plasma samples from subjects in the PK database that were below the limit of detection of the assay, a mixtureof-models approach was used, whereby the Pop PK model classified subjects as "absorbers" or "non-absorbers." In the final model that provided the best fit of all the data, 71 subjects were classified as "absorbers" and 37 were classified as "non-absorbers." Values below the limit of quantitation were assigned a value of $1 / 2$ the LLOQ. Then, a full covariate model was developed using pre-specified covariates, including age, body mass index, weight, sex, race, and formulation. The full model underwent backward elimination to identify the most important variables in a stepwise manner, leading to a parsimonious final model that contained covariates that were statistically significant. After model development and qualification were complete, the final Pop PK model was used to generate metrics of exposure $\left(C_{\max }, \mathrm{AUC}\right)$ for patients who had been randomized to receive active treatment. For subjects in the "absorber" subpopulation, peak concentration values $\left(C_{\max }\right)$ were taken directly from the observed data and AUC was calculated as the administered dose divided by clearance. For the "non-absorber" subpopulation, $C_{\max }$ was set to the LLOQ, and the AUC was computed assuming concentrations over the dose interval were at LLOQ. These exposure metrics were then used for subsequent exploration of exposure-response relationships in terms of AEs and efficacy. Additional methodological details are included in the ESM.

\subsubsection{Safety and Efficacy}

Adverse events were monitored throughout the phase II studies. Patients were instructed to inform the investigator and/ or study staff of any AE, and at each visit, patients were asked about AEs in a non-specific manner using open-ended questions so as not to bias the response (e.g., "How have you been since the last visit?"). The severity of an AE was designated as mild, moderate, or severe by the investigator based on the subject's description and, when applicable, by direct observation on the part of the investigator. Efficacy was assessed at each visit by gravimetric measurement of sweat production and HDSS.

For the Pop PK analysis, exposure metrics were used to assess the relationship between topical glycopyrronium pharmacokinetics and anticholinergic-related AEs or efficacy. Patient data from the vehicle treatment arms were included in these analyses (exposure metrics for vehicle patients were set to 0). The Pop PK-AE model database included information on glycopyrronium exposure and the most severe grade of AEs that could be due to anticholinergic activity. The AEs included in the analysis were dry mouth, vision blurred, urinary retention, dry eye, mydriasis, urinary hesitation, urine flow decreased, dry tongue, constipation, nasal dryness, and vulvovaginal dryness. Level of severity was captured using a numerical code ( 0 for no events, 1 for mild, 2 for moderate, and 3 for severe). For the analysis of efficacy, a Pop PK-pharmacodynamic model database included information on glycopyrronium exposure 
and multiple assessments of gravimetric sweat production and HDSS scores.

\section{Results}

\subsection{Phase I PK Study}

\subsubsection{Subjects and Samples}

A total of 49 subjects were enrolled, 31 in the GT arm (11 adult and 20 pediatric subjects) and 18 in the oral glycopyrrolate arm (all were adults); all subjects completed the study (Table 1). Demographics and baseline characteristics are summarized in Table 1.

Following application of GT, plasma glycopyrronium concentrations were quantifiable in most patients up to the last scheduled timepoint of $24 \mathrm{~h}$ in adults and $6 \mathrm{~h}$ in pediatric patients. Following oral glycopyrrolate administration, plasma glycopyrronium concentrations were quantifiable in all subjects for the entire sampling period of up to 24 h. A total of 15 concentration values (out of a total of 646 planned samples in the GT arm) were excluded from seven subjects in the GT arm (three adult; four pediatric) because the values were greater than 3 standard deviations from the mean; 8 of these 15 values were from one pediatric subject on day 1 . No concentration values were excluded for the oral glycopyrrolate arm.

\subsubsection{Pharmacokinetics}

There was no evidence of accumulation of GT when comparing PK parameters for day 1 (Table 1 of the ESM) and day 5 (Table 2). On day $1, C_{\max }$ and AUC values for glycopyrronium were similar in adults receiving GT or oral glycopyrrolate and lower in pediatric patients receiving GT (Table 1 of the ESM). On day 5, the $C_{\max }$ and AUC values
Table 1 Phase I study: subject disposition and baseline characteristics

\begin{tabular}{|c|c|c|c|}
\hline Characteristics & $\begin{array}{l}\text { Topical glycopyrro- } \\
\text { nium tosylate } \\
\text { Adult } n=11\end{array}$ & $\begin{array}{l}\text { Topical glycopyrro- } \\
\text { nium tosylate } \\
\text { Pediatric } n=20\end{array}$ & $\begin{array}{l}\text { Oral glycopyrrolate } \\
\text { Adult } n=18\end{array}$ \\
\hline \multicolumn{4}{|l|}{ Subjects } \\
\hline Enrolled/completed & $11 / 11$ & $20 / 20$ & $18 / 18$ \\
\hline Safety population $^{\mathrm{a}}$ & 11 & 20 & 18 \\
\hline PK evaluable population ${ }^{\mathrm{b}}$ & 11 & $20^{\mathrm{c}}$ & 18 \\
\hline \multicolumn{4}{|l|}{ Age } \\
\hline Mean (SD) & $26.0(8.9)$ & $14.8(1.6)$ & $44.0(10.4)$ \\
\hline Median (min, max) & $23.0(18,49)$ & $15.0(10,17)$ & $46.5(18,58)$ \\
\hline \multicolumn{4}{|l|}{$\operatorname{Sex}[n(\%)]$} \\
\hline Male & $4(36.4)$ & $7(35.0)$ & $16(88.9)$ \\
\hline Female & $7(63.6)$ & $13(65.0)$ & $2(11.1)$ \\
\hline \multicolumn{4}{|l|}{$\operatorname{Race}[n(\%)]$} \\
\hline White & $9(81.8)$ & $13(65.0)$ & $9(50.0)$ \\
\hline Black or African American & $2(18.2)$ & $7(35.0)$ & $8(44.4)$ \\
\hline Other & 0 & 0 & $1(5.6)$ \\
\hline \multicolumn{4}{|l|}{ Ethnicity $[n(\%)]$} \\
\hline Hispanic or Latino & $2(18.2)$ & 0 & $2(11.1)$ \\
\hline Not Hispanic or Latino & $9(81.8)$ & $20(100.0)$ & $16(88.9)$ \\
\hline \multicolumn{4}{|l|}{ Weight (kg) } \\
\hline Mean (SD) & $87.8(27.7)$ & $67.2(16.9)$ & $80.2(9.8)$ \\
\hline Median (min, max) & $79.5(61.7,156.9)$ & $63.6(46.3,108.0)$ & $78.8(66.7,98.8)$ \\
\hline \multicolumn{4}{|l|}{$\mathrm{BMI}\left(\mathrm{kg} / \mathrm{m}^{2}\right)$} \\
\hline Mean (SD) & $29.4(6.3)$ & $23.9(5.6)$ & $27.2(2.4)$ \\
\hline Median (min, max) & $29.5(21.9,40.0)$ & $22.2(18.6,40.8)$ & $27.8(23.5,30.8)$ \\
\hline
\end{tabular}

$B M I$ body mass index, $\max$ maximum, min minimum, $P K$ pharmacokinetic, $S D$ standard deviation

${ }^{a}$ Subjects who were enrolled and received $\geq 1$ confirmed dose of the study drug

${ }^{\mathrm{b}}$ Subjects who received the study drug and had $\geq 1 \mathrm{PK}$ sample collected

${ }^{\mathrm{c}} 19$ and 20 subjects, respectively, were included in the PK evaluable population for days 1 and 5 
Table 2 Phase I study: pharmacokinetic (PK) findings for topical glycopyrronium (adult and pediatric patients) and oral glycopyrrolate (adults only)

\begin{tabular}{|c|c|c|c|c|c|}
\hline \multirow[t]{2}{*}{ PK parameter } & \multirow{2}{*}{$\begin{array}{l}\text { Topical glycopyrro- } \\
\text { nium tosylate } \\
\text { Adult } \\
2.4 \%\end{array}$} & \multirow{2}{*}{$\begin{array}{l}\text { Topical glycopyrro- } \\
\text { nium tosylate } \\
\text { Pediatric } \\
2.4 \%\end{array}$} & \multicolumn{3}{|c|}{$\begin{array}{l}\text { Oral glycopyrrolate } \\
\text { Adult }\end{array}$} \\
\hline & & & $1 \mathrm{mg} / \mathrm{q} 8 \mathrm{~h}$ & $2 \mathrm{mg} / \mathrm{q} 8 \mathrm{~h}$ & $3 \mathrm{mg} / \mathrm{q} 8 \mathrm{~h}$ \\
\hline & Day 5 of 5 & Day 5 of 5 & Day 5 of 15 & Day 10 of 15 & Day 15 of 15 \\
\hline$C_{\max }(\mathrm{ng} / \mathrm{mL})$ & $n=11$ & $n=20$ & $n=18$ & $n=18$ & $n=18$ \\
\hline Mean \pm SD & $0.08 \pm 0.04$ & $0.07 \pm 0.06$ & $0.15 \pm 0.12$ & $0.23 \pm 0.11$ & $0.38 \pm 0.19$ \\
\hline Median (min, max) & $0.08(0.02,0.15)$ & $0.04(0,0.25)$ & $0.12(0.05,0.54)$ & $0.22(0.10,0.42)$ & $0.34(0.13,0.89)$ \\
\hline $\operatorname{AUC}_{0-6 \mathrm{~h}}(\mathrm{ng} \mathrm{h} / \mathrm{mL})$ & $n=10$ & $n=11$ & $n=18$ & $n=8$ & $n=18$ \\
\hline Mean \pm SD & $0.20 \pm 0.14$ & $0.18 \pm 0.13$ & $0.61 \pm 0.44$ & $0.98 \pm 0.41$ & $1.57 \pm 0.64$ \\
\hline Median (min, max) & $0.14(0.09,0.57)$ & $0.15(0,0.50)$ & $0.49(0.20,2.01)$ & $1.03(0.44,1.70)$ & $1.51(0.64,2.85)$ \\
\hline $\mathrm{AUC}_{0-24 \mathrm{~h}}(\mathrm{ng} \mathrm{h} / \mathrm{mL})$ & $n=7$ & Not determined ${ }^{\mathrm{b}}$ & $n=18$ & $n=18$ & $n=18$ \\
\hline Mean \pm SD & $0.88 \pm 0.57$ & Not determined ${ }^{\mathrm{b}}$ & $2.12 \pm 1.47$ & $3.50 \pm 1.5$ & $5.50 \pm 2.19$ \\
\hline Median (min, max) & $0.79(0.37,1.93)$ & Not determined ${ }^{\mathrm{b}}$ & $1.74(0.70,6.84)$ & $3.53(1.49,5.94)$ & $5.34(2.21,9.81)$ \\
\hline$T_{\max }(\mathrm{h})$ & $n=11$ & $n=19$ & $n=18$ & $n=18$ & $n=18$ \\
\hline Mean \pm SD & $3.18 \pm 4.41$ & $1.76 \pm 1.53$ & $2.39 \pm 0.99$ & $2.86 \pm 1.60$ & $2.19 \pm 1.10$ \\
\hline Median (min, max) & $1.0(0,10)$ & $1.50(0,6)$ & $2.50(1.00,4.00)$ & $2.75(0.50,6.00)$ & $2.00(0.50,4.00)$ \\
\hline$T_{1 / 2}(\mathrm{~h})$ & & & $n=14$ & $n=11$ & $n=12$ \\
\hline Mean \pm SD & Not determined ${ }^{\mathrm{c}}$ & Not determined ${ }^{c}$ & $2.59 \pm 0.65$ & $2.80 \pm 0.48$ & $2.76 \pm 0.88$ \\
\hline Median $(\min , \max )$ & Not determined ${ }^{c}$ & Not determined $^{\mathrm{c}}$ & $2.64(1.78,4.16)$ & $2.92(1.98,3.60)$ & $2.53(1.62,4.52)$ \\
\hline
\end{tabular}

The PK evaluable population included subjects who received study drug and had $\geq 1 \mathrm{PK}$ sample collected

$A U C$ area under the plasma concentration vs time curve, $C_{\max }$ maximum plasma concentration, $h$ hours, $\max$ maximum, min minimum, $S D$ standard deviation, $T_{1 / 2}$ elimination (terminal) half-life, $T_{\max }$ time to maximum plasma concentration, $q 8 \mathrm{~h}$ every $8 \mathrm{~h}$

${ }^{\text {a }}$ Fasting

${ }^{\mathrm{b}}$ Pediatric samples were only collected up to $6 \mathrm{~h}$ post-dose per guidelines on safe blood sampling; therefore, $\mathrm{AUC}_{0-24 \mathrm{~h}}$ could not be determined

${ }^{\mathrm{c}}$ A clear terminal elimination phase was not evident following topical glycopyrronium tosylate administration owing to a lack of concentrations above the lower limit of quantitation; thus, no half-life could be determined in adult and pediatric patients

for glycopyrronium calculated for both adult and pediatric patients who received GT were lower than those observed on days 5,10 , or 15 in adults who received oral glycopyrrolate (Table 2). In the comparisons of relative glycopyrronium exposure for GT (day 5) vs oral glycopyrrolate (days 5, 10, or 15 ), none of the $90 \%$ CIs of the geometric mean ratios included the value of 1 (Table 3 ).

Following GT treatment, $C_{\max }$ and AUC from 0 to $6 \mathrm{~h}$ for glycopyrronium were similar in adult and pediatric patients (blood samples for pediatric patients were only taken up to 6 hours post-dose; therefore, $\mathrm{AUC}_{0-24 \mathrm{~h}}$ could not be determined; Table 2). Exposure to glycopyrronium in pediatric patients was nominally lower than that in adult patients, but the $90 \%$ CIs of the geometric mean ratios were inclusive of 1 (Table 3).

Median $T_{\max }$ values were $1.0-1.5 \mathrm{~h}$ in adult and pediatric patients following GT treatment, while the median $T_{\max }$ for oral glycopyrrolate was $2.0-2.75 \mathrm{~h}$ (Table 2 ). When looking at mean values, it is worth noting that the mean $T_{\max }$ value for GT in adults was higher than the mean $T_{\max }$ value for oral glycopyrrolate. The standard deviation was also large indicating high variability, which may indicate inter-individual differences in the rate of absorption with a skewing of the data because of longer $T_{\max }$ values in some subjects.

\subsubsection{Safety}

Treatment-emergent AEs are summarized in Table 4. No anticholinergic-related TEAEs occurred following GT treatment, while those occurring with oral glycopyrrolate treatment included dry mouth $(16.7 \%)$ and nasal dryness (5.6\%). All TEAEs were deemed mild in severity. Of the seven subjects in the GT arm who had concentration values excluded because they were $>3$ standard deviations from the mean, six of seven had no AEs reported during the trial; one of the adult subjects had an AE of mild headache on day 2 (the concentration value excluded was $8 \mathrm{~h}$ post-dose on day 1). GT was well tolerated locally. Most patients treated with GT had no local skin reactions (9/11 adults and 14/20 pediatric patients), and local skin reactions that were reported were all mild. 
Table 3 Comparison of relative exposure

\begin{tabular}{|c|c|c|c|c|}
\hline \multirow[t]{2}{*}{ Comparison } & \multicolumn{2}{|l|}{$C_{\max }(\mathrm{ng} / \mathrm{mL})$} & \multirow{2}{*}{$\begin{array}{l}\text { AUC }_{0-6 h}(\mathrm{ng} \mathrm{h} / \mathrm{mL}) \\
\text { Pediatric }\end{array}$} & \multirow{2}{*}{$\begin{array}{l}\mathrm{AUC}_{0-24 \mathrm{~h}}(\mathrm{ng} \mathrm{h} / \mathrm{mL}) \\
\text { Adult }\end{array}$} \\
\hline & Adult & Pediatric & & \\
\hline \multicolumn{5}{|l|}{ GT pediatric day 5 vs GT adult day 5} \\
\hline $\begin{array}{l}\text { Geometric mean ratio }(90 \% \mathrm{CI}) \text { for pediatric [test]/adult [refer- } \\
\text { ence] }\end{array}$ & & $0.64(0.37,1.10)$ & $0.87(0.54,1.4)$ & Not determined ${ }^{\mathrm{a}}$ \\
\hline \multicolumn{5}{|l|}{ GT day 5 vs oral glycopyrrolate day 5} \\
\hline $\begin{array}{l}\text { Geometric mean ratio }(90 \% \mathrm{CI}) \text { for GT [test]/oral glycopyr- } \\
\text { rolate [reference] }\end{array}$ & $0.55(0.38,0.80)$ & $0.35(0.22,0.55)$ & $0.28(0.19,0.43)$ & $0.41(0.26,0.65)$ \\
\hline \multicolumn{5}{|l|}{ GT day 5 vs oral glycopyrrolate day 10} \\
\hline $\begin{array}{l}\text { Geometric mean ratio }(90 \% \mathrm{CI}) \text { for GT [test]/oral glycopyr- } \\
\text { rolate [reference] }\end{array}$ & $0.34(0.25,0.48)$ & $0.22(0.14,0.34)$ & $0.16(0.11,0.23)$ & $0.23(0.16,0.34)$ \\
\hline \multicolumn{5}{|l|}{ GT day 5 vs oral glycopyrrolate day 15} \\
\hline $\begin{array}{l}\text { Geometric mean ratio }(90 \% \mathrm{CI}) \text { for GT [test]/oral glycopyr- } \\
\text { rolate [reference] }\end{array}$ & $0.21(0.15,0.29)$ & $0.13(0.09,0.20)$ & $0.10(0.07,0.15)$ & $0.15(0.10,0.21)$ \\
\hline
\end{tabular}

$A U C_{0-6 h}$ area under the plasma concentration vs time curve from time 0 to 6 hours, $A U C_{0-24 h}$ area under the plasma concentration vs time curve from time 0 to 24 hours, $C I$ confidence interval, $G T$ glycopyrronium tosylate

${ }^{a}$ Pediatric samples were only collected up to $6 \mathrm{~h}$ post-dose per guidelines on safe blood sampling; therefore, $\mathrm{AUC}_{0-24 \mathrm{~h}}$ could not be determined

Table 4 Phase I study: treatment-emergent adverse events (TEAEs)

\begin{tabular}{lllll}
\hline & \multicolumn{2}{l}{ Topical glycopyrronium tosylate $N=31$} & $\begin{array}{l}\text { Oral glyco- } \\
\text { pyrrolate } n \\
=\end{array}$ \\
\cline { 2 - 5 } & Adult $n=11$ & Pediatric $n=20$ & Total $n=31$ & $\begin{array}{l}\text { Ply } \\
\text { TEAEs }[n(\%)]\end{array}$ \\
Dry mouth & $2(18.2)$ & $3(15.0)$ & $5(16.1)$ & $7(38.9)$ \\
Headache & 0 & 0 & 0 & $3(16.7)$ \\
Nocturia & $2(18.2)$ & $2(10.0)$ & $4(12.9)$ & $1(5.6)$ \\
Chest pain & 0 & 0 & 0 & $2(11.1)$ \\
Hypoesthesia & 0 & 0 & 0 & $1(5.6)$ \\
Nasal dryness & 0 & 0 & 0 & $1(5.6)$ \\
Cough & 0 & 0 & 0 & $1(5.6)$ \\
Laceration & 0 & $1(5.0)$ & $1(3.2)$ & 0 \\
Rhinorrhea & 0 & $1(5.0)$ & $1(3.2)$ & 0 \\
TEAE by severity $[n(\%)]$ & 0 & $1(5.0)$ & $1(3.2)$ & 0 \\
Mild & & & & $7(38.9)$ \\
Moderate & $2(18.2)$ & $3(15.0)$ & $5(16.1)$ & 0 \\
Severe & 0 & 0 & 0 & 0 \\
Treatment-related TEAE $[n(\%)]$ & 0 & 0 & 0 & $5(27.8)$ \\
Serious TEAEs $[n(\%)]$ & 0 & 0 & 0 & 0 \\
TEAE leading to discontinuation $[n(\%)]$ & 0 & 0 & 0 & 0 \\
\hline
\end{tabular}

\subsection{Population PK Study}

\subsubsection{Subjects and Samples}

For the Pop PK model development, 1168 PK samples from the active treatment groups were initially available. A total of 109 pre-dose samples were excluded as were 74 post-dose samples because of no results reported $(n=59)$, observations missing ( $n=6)$, and conditional weighted residual $>5(n=9)$, resulting in the inclusion of $985 \mathrm{PK}$ samples from 108 patients (mean age 32.6 years, $55.6 \%$ male) and AE/efficacy data for 137 patients ( $n=108$ glycopyrronium, $n=29$ vehicle; mean age 32.8 years, $53.3 \%$ male) from the phase II studies (Table 2 of the ESM and Table 5). A total of $57.9 \%$ of the PK samples (676/1168) were below the limit of quantitation. 


\subsubsection{Population Pharmacokinetics}

In the DRM04-HH01 and DRM04-HH02 studies, there was no evidence of accumulation with repeated dosing (data not shown), and the estimated bioavailability (0.5\%) was low in subjects who absorbed the drug. Following model testing, $C_{\max }$ was found to be predictive of the probability of anticholinergic AE frequency and severity. As shown in Fig. 2, the probability of anticholinergic AEs increased with higher glycopyrronium concentrations; however, the mean

Table 5 Population pharmacokinetic (Pop PK) study: patient demographics and characteristics

\begin{tabular}{lll}
\hline $\begin{array}{l}\text { Demographics and } \\
\text { characteristics }\end{array}$ & $\begin{array}{l}\text { Pop PK database } N= \\
108\end{array}$ & $\begin{array}{l}\text { Pop PK AE and PD } \\
\text { database } N=137\end{array}$ \\
\hline Age & $32.6(11.6)$ & $32.8(11.2)$ \\
Mean (SD) & $30.0(18,72)$ \\
Median (min, max) & $29.5(18,72)$ & $73(53.3)$ \\
Sex $[n(\%)]$ & $60(55.6)$ & $64(46.7)$ \\
Male & $48(44.4)$ & $121(88.3)$ \\
Female & & $12(8.8)$ \\
Race $[n(\%)]$ & $94(87.0)$ & $1(0.7)$ \\
Caucasian & $10(9.3)$ & $3(2.2)$ \\
Black & $1(0.9)$ & $83.4(22.2)$ \\
Asian & $3(2.8)$ & $80.7(45.4,153)$ \\
Other & & \\
Weight $(\mathrm{kg})$ & $84.1(22.6)$ & $28.0(6.0)$ \\
Mean $(\mathrm{SD})$ & $82.1(45.4,152)$ & $27.2(17.2,46.8)$ \\
Median (min, max) & & \\
BMI (kg/m $\left.{ }^{2}\right)$ & $28.4(6.2)$ &
\end{tabular}

$A E$ adverse event, $B M I$ body mass index, $\max$ maximum, $\min$ minimum, $P D$ pharmacodynamic, $S D$ standard deviation
$(0.0985 \mu \mathrm{g} / \mathrm{L}$ [standard deviation 0.213]) and median (0.026 $\mu \mathrm{g} / \mathrm{L}$ [minimum, maximum $[0,1.67]) C_{\max }$ values were low. Figure 3 shows the probability of differing severities of anticholinergic AEs. Mild AEs tended to occur at low concentrations, while the probability of moderate/severe AEs did not become appreciable (over $20 \%$ ) until $C_{\max }$ values of approximately $0.2 \mu \mathrm{g} / \mathrm{L}$; however, most patients in the active treatment arms (85.2\% [92/108]) had a glycopyrronium $C_{\max }$ value less than $0.2 \mu \mathrm{g} / \mathrm{L}$ (Fig. 3). For efficacy, gravimetric measurement of sweat and HDSS did not correlate with exposure. For example, a change in gravimetric score or HDSS grade at week 4 vs AUC, $C_{\max }$, and dose strength showed no or only weak trends (Figs. 2, 3 of the ESM).

\section{Discussion}

In the phase I study, PK parameters were similar between adult and pediatric patients treated with a GT $2.4 \%$ cloth, and systemic absorption was lower with GT vs oral glycopyrrolate. No anticholinergic-related TEAEs occurred with GT in the phase I study, whereas dry mouth and nasal dryness occurred with oral glycopyrrolate. In the Pop PK study, exposure-response models indicated that the frequency and severity of anticholinergic-related AEs increases with higher glycopyrronium concentrations, and the low mean and median glycopyrronium $C_{\max }$ values indicate a favorable AE profile with GT administration.

When interpreting the results of the phase I study, it should be noted that GT was applied by study staff, not by patients, which may have reduced the potential for unintended systemic exposure from incorrect application and may account for the lack of anticholinergic TEAEs observed in the GT group. As noted in the Pop PK analysis, anticholinergic AEs occurred with patient-applied GT in the phase
Fig. 2 Population pharmacokinetic study: probability of anticholinergic adverse events (frequency). Shading denotes the $95 \%$ confidence interval; patients randomized to vehicle were assigned a value of " 0 " for glycopyrronium exposure. $C I$ confidence interval, $C_{\max }$ maximum plasma concentration

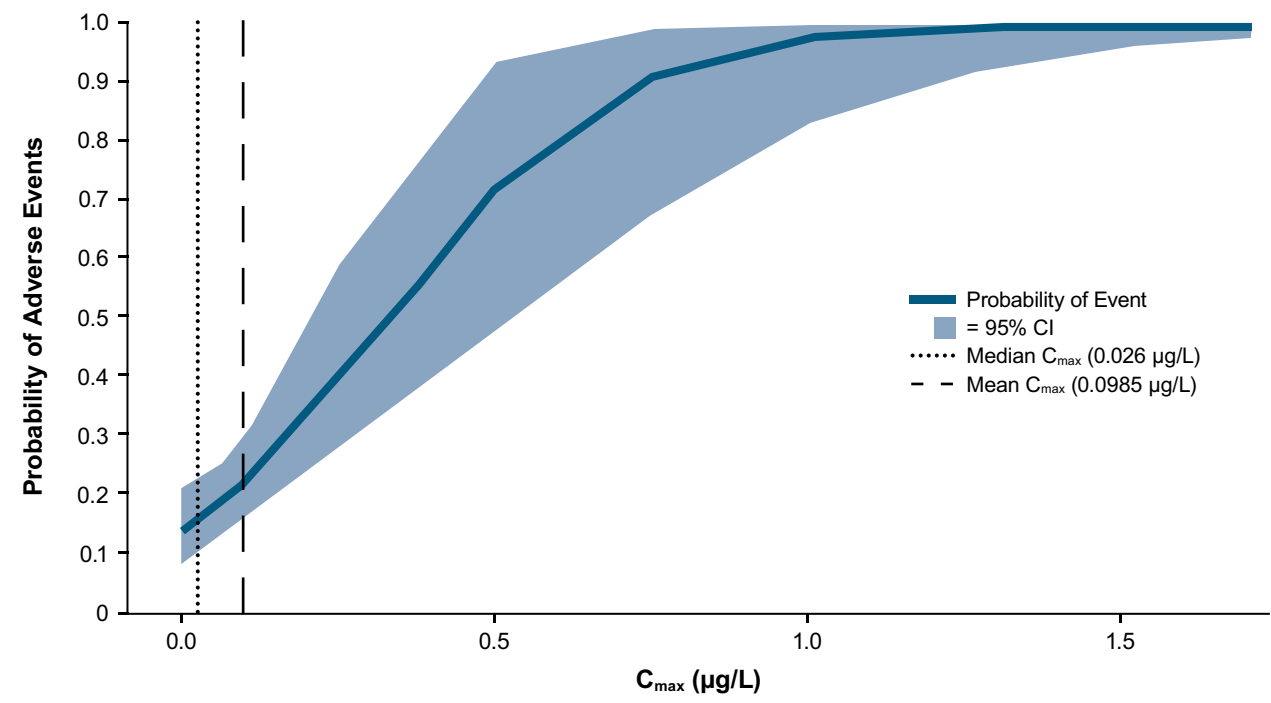


Fig. 3 Population pharmacokinetic study: probability of anticholinergic adverse events (severity). Shading denotes the 95\% confidence interval. $C I$ confidence interval, $C_{\max }$ maximum plasma concentration

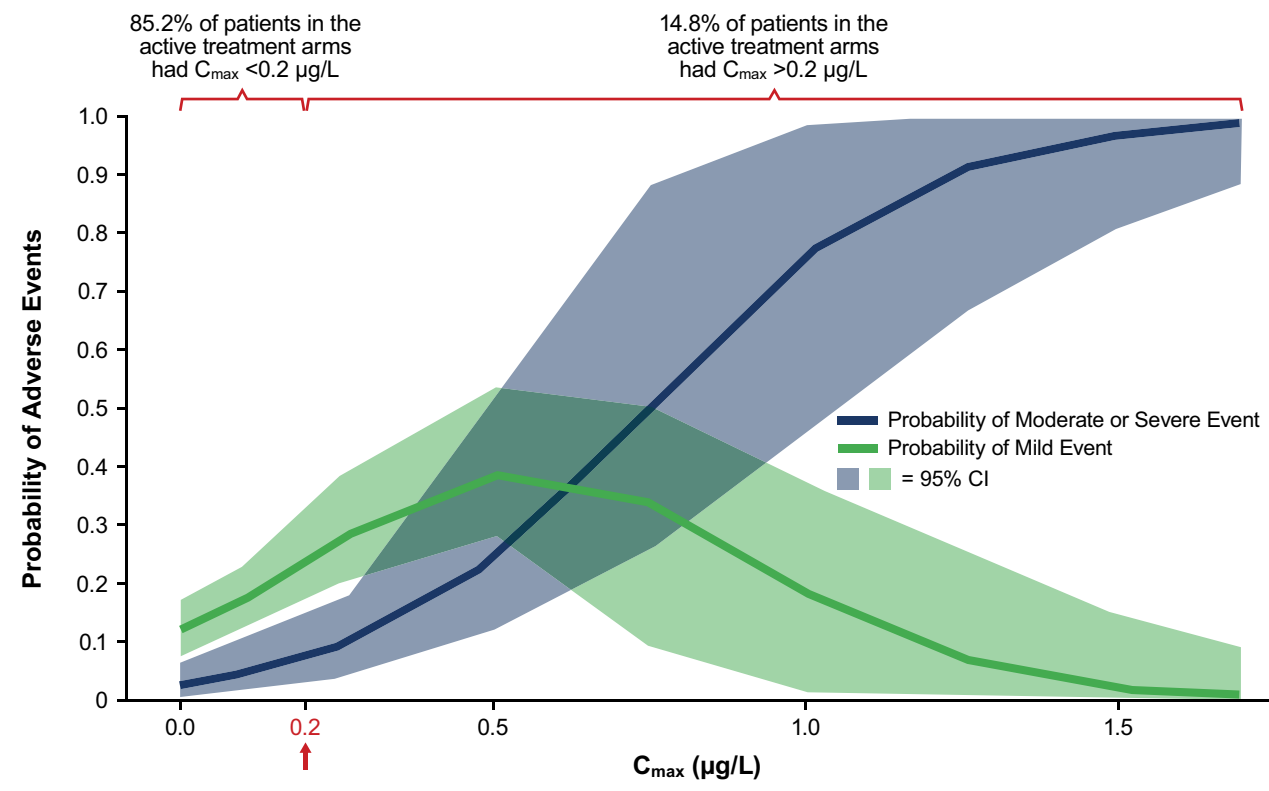

II, double-blind studies, and they also were reported in the phase III, double-blind studies where GT was applied by patients [10]. Consistent with the results reported here, most TEAEs in the phase III, double-blind trials were mild and infrequently led to discontinuation, including in pediatric patients $[10,14]$. Further features of the phase I study that may limit the generalizability of the results include the small sample size of the study population, the different subject populations treated with each drug, and the large $90 \%$ CIs of the geometric mean ratios for $C_{\max }$ and AUC when comparing adult and pediatric GT exposure.

The pharmacokinetic results observed in our phase I study for oral glycopyrrolate solution $(1,2$, or $3 \mathrm{mg} / \mathrm{kg} / 8$ h) in healthy fasting adults are similar to results reported in the literature for oral glycopyrrolate solution $(2 \mathrm{mg} / \mathrm{kg} /$ day $)$ in healthy fasting adults $\left(C_{\max }\right.$ of $0.318 \mathrm{ng} / \mathrm{mL}, \mathrm{AUC}_{0-24}$ of $1.74 \mathrm{ng} \cdot \mathrm{h} / \mathrm{mL}, T_{\max }$ of $3.1 \mathrm{~h}$, and elimination (terminal) half-life of $3.0 \mathrm{~h}$ ) $[16,20]$. At present, there is a dearth of publicly available pharmacokinetic data for topical formulations containing glycopyrronium that are under development; a $1 \%$ glycopyrronium bromide cream has a reported $C_{\text {max }}$ of $0.024 \mathrm{ng} / \mathrm{mL}$ and $\mathrm{AUC}_{0-24}$ of $0.107 \mathrm{ng} \cdot \mathrm{h} / \mathrm{mL}$ [20], but the primary study has not been reported and, therefore, sufficient detail is not available to make informed comparative statements to GT.

In the Pop PK analysis, most patients had exposure levels much lower than those that correlated with an increased probability of a moderate or severe AE. Variability in exposure level may be owing to patient characteristics or application technique or a combination of the two. For efficacy, the probability of having an efficacious result was not related to either $C_{\max }$ or AUC, which suggests that efficacy may be mediated through a local effect of topical administration. In contrast, systemic exposure did account for the occurrence of anticholinergic AEs. Features of the Pop PK analysis that may limit the generalizability of the results include the lack of pediatric patients in the study population, the moderate size of the study population, and the fact that these data are derived from assumptions used in modeling. In addition, the model also does not provide information on exposure-response relationships with long-term use of GT, though in a long-term extension trial of GT, most patients experiencing TEAEs had mild or moderate events, including pediatric patients $[11,21]$.

Overall, the phase I and Pop PK studies indicate that there is limited absorption and a low risk of anticholinergic AEs with proper GT administration, which is consistent with clinical observations to date and underscores the importance of patient instructions for use, which specify to wipe each underarm once with the same cloth, wash hands with soap and water immediately after discarding the used cloth, and avoid ocular contact to prevent blurred vision and temporary dilation of the pupils [13].

Acknowledgements Medical writing support for the article was provided by Prescott Medical Communications Group (Chicago, IL, USA), with financial support from Dermira, Inc., a wholly owned subsidiary of Eli Lilly and Company.

\section{Declarations}

Funding The studies described in this article were funded by Dermira, Inc., a wholly owned subsidiary of Eli Lilly and Company.

Conflict of interest David M. Pariser was an investigator and consultant for Dermira, Inc., a wholly owned subsidiary of Eli Lilly and Company, and received writing assistance. Edward L. Lain has re- 
ceived payment from the sponsor for work as a principal investigator, advisory board member, speaker, and consultant, but did not receive compensation for work related to the preparation and publication of this article. Richard D. Mamelok is a paid consultant to Dermira, Inc., a wholly owned subsidiary of Eli Lilly and Company, and received financial support for reviewing data and for reviewing the manuscript. Janice Drew is a full-time employee and receives a salary from the sponsor of the study, Dermira, Inc., a wholly owned subsidiary of Eli Lilly and Company. Diane R. Mould is the president of Projections Research Inc., a consulting company for the pharmaceutical industry and was a paid consultant of Dermira, Inc., a wholly owned subsidiary of Eli Lilly and Company for the conduct of the data analysis.

Ethics approval For the phase I and II clinical studies described in the article, the protocols, informed consent forms, and assent forms for subjects under legal adult age (phase I study only) were submitted to an institutional review board or independent ethics committee for review and approval before the start of the study. The studies were conducted in compliance with the principles of the International Conference on Harmonisation (E6 1997) Good Clinical Practice, US Food and Drug Administration guidelines, and the Declaration of Helsinki, 1964 (as amended in Edinburgh [2000]).

Consent to participate For the phase I and II clinical studies described in the article, informed consent was obtained.

Consent for publication All authors consent to the publication of the article.

Availability of data and material Dermira, Inc., a wholly owned subsidiary of Eli Lilly and Company, shares data related to these trials in peer-reviewed journals, medical congresses, and ClinicalTrials.gov. Individual patient data are not publicly available.

Code availability Not applicable.

Authors' contributions JD, DRM, and RDM had access to all of the data in the study and take responsibility for the integrity of the data and the accuracy of the data analysis. JD, DRM, and RDM provided the concept and design for the study. All authors were responsible for the acquisition, analysis, or interpretation of the data and the drafting and critical revision of the manuscript for important intellectual content. DRM and RDM conducted the data analysis.

Open Access This article is licensed under a Creative Commons Attribution-NonCommercial 4.0 International License, which permits any non-commercial use, sharing, adaptation, distribution and reproduction in any medium or format, as long as you give appropriate credit to the original author(s) and the source, provide a link to the Creative Commons licence, and indicate if changes were made. The images or other third party material in this article are included in the article's Creative Commons licence, unless indicated otherwise in a credit line to the material. If material is not included in the article's Creative Commons licence and your intended use is not permitted by statutory regulation or exceeds the permitted use, you will need to obtain permission directly from the copyright holder. To view a copy of this licence, visit http://creativecommons.org/licenses/by-nc/4.0/.

\section{References}

1. Moraites E, Vaughn OA, Hill S. Incidence and prevalence of hyperhidrosis. Dermatol Clin. 2014;32(4):457-65. https://doi. org/10.1016/j.det.2014.06.006.
2. Benson RA, Palin R, Holt PJ, Loftus IM. Diagnosis and management of hyperhidrosis. BMJ. 2013;347:f6800. https://doi. org/10.1136/bmj.f6800.

3. Nawrocki S, Cha J. The etiology, diagnosis, and management of hyperhidrosis: a comprehensive review: etiology and clinical work-up. J Am Acad Dermatol. 2019;81(3):657-66. https://doi. org/10.1016/j.jaad.2018.12.071.

4. Doolittle J, Walker P, Mills T, Thurston J. Hyperhidrosis: an update on prevalence and severity in the United States. Arch Dermatol Res. 2016;308(10):743-9. https://doi.org/10.1007/s00403-016-1697-9.

5. Grabell DA, Hebert AA. Current and emerging medical therapies for primary hyperhidrosis. Dermatol Ther (Heidelb). 2017;7(1):25-36. https://doi.org/10.1007/s13555-016-0148-z.

6. Bahar R, Zhou P, Liu Y, Huang Y, Phillips A, Lee TK, et al. The prevalence of anxiety and depression in patients with or without hyperhidrosis (HH). J Am Acad Dermatol. 2016;75(6):1126-33. https://doi.org/10.1016/j.jaad.2016.07.001.

7. Kamudoni P, Mueller B, Halford J, Schouveller A, Stacey B, Salek MS. The impact of hyperhidrosis on patients' daily life and quality of life: a qualitative investigation. Health Qual Life Outcomes. 2017;15(1):121. https://doi.org/10.1186/s 1295 5-017-0693-x.

8. Nawrocki S, Cha J. The etiology, diagnosis, and management of hyperhidrosis: a comprehensive review: therapeutic options. J Am Acad Dermatol. 2019;81(3):669-80. https://doi.org/10.1016/j. jaad.2018.11.066.

9. Lamb YN. Topical glycopyrronium tosylate in primary axillary hyperhidrosis: a profile of its use. Clin Drug Investig. 2019;39(11):1141-7. https://doi.org/10.1007/s40261-019-00853 -X.

10. Glaser DA, Hebert AA, Nast A, Werschler WP, Green L, Mamelok R, et al. Topical glycopyrronium tosylate for the treatment of primary axillary hyperhidrosis: results from the ATMOS- 1 and ATMOS- 2 phase 3 randomized controlled trials. J Am Acad Dermatol. 2019;80(1):128-38. https://doi.org/10.1016/j. jaad.2018.07.002.

11. Glaser DA, Hebert AA, Nast A, Werschler WP, Green L, Mamelok $\mathrm{RD}$, et al. A 44-week open-label study evaluating safety and efficacy of typical glycopyrronium tosylate in patients with primary axillary hyperhidrosis. Am J Clin Dermatol. 2019;20(4):593-604. https://doi.org/10.1007/s40257-019-00446-6.

12. Pariser DM, Hebert AA, Drew J, Quiring J, Gopalan R, Glaser DA. Topical glycopyrronium tosylate for the treatment of primary axillary hyperhidrosis: patient-reported outcomes from the ATMOS- 1 and ATMOS-2 phase III randomized controlled trials. Am J Clin Dermatol. 2019;20(1):135-45. https://doi.org/10.1007/ s40257-018-0395-0.

13. Qbrexza $^{\circledR}$ (glycopyrronium) cloth, $2.4 \%$, for topical use [prescribing information]. Menlo Park (CA): Dermira Inc., 2018.

14. Hebert AA, Glaser DA, Green L, Werschler WP, Forsha DW, Drew J, et al. Glycopyrronium tosylate in pediatric primary axillary hyperhidrosis: post hoc analysis of efficacy and safety findings by age from two phase three randomized controlled trials. Pediatr Dermatol. 2019;36(1):89-99. https://doi.org/10.1111/ pde. 13723.

15. International Hyperhidrosis Society. Primary axillary hyperhidrosis. 2018. https://www.sweathelp.org/pdf/Axillary_IHhS_Algor ithm_2018.pdf. Accessed 03 Apr 2020.

16. Cuvposa ${ }^{\circledR}$ (glycopyrrolate) oral solution [prescribing information]. Raleigh (NC): Merz Pharmaceuticals, LLC, 2015.

17. Nelson LM, DiBenedetti D, Pariser DM, Glaser DA, Hebert AA, Hofland H, et al. Development and validation of the Axillary Sweating Daily Diary: a patient-reported outcome measure to assess axillary sweating severity. J Patient Rep Outcomes. 2019;3(1):59. https://doi.org/10.1186/s41687-019-0148-8. 
18. National Center for Biotechnology Information. PubChem database. Glycopyrrolate, CID=11693. https://pubchem.ncbi.nlm.nih. gov/compound/11693. Accessed 17 June 2020.

19. National Center for Biotechnology Information. PubChem database. Glycopyrronium tosylate, $\mathrm{CID}=112500546$. https://pubch em.ncbi.nlm.nih.gov/compound/112500546. Accessed 17 Jun 2020.

20. Chabicovsky M, Winkler S, Soeberdt M, Kilic A, Masur C, Abels C. Pharmacology, toxicology and clinical safety of glycopyrrolate. Toxicol Appl Pharmacol. 2019;370:154-69. https://doi. org/10.1016/j.taap.2019.03.016.
21. Hebert AA, Glaser DA, Green L, Hull C, Cather J, Drew J, et al. Long-term efficacy and safety of topical glycopyrronium tosylate for the treatment of primary axillary hyperhidrosis: post hoc pediatric subgroup analysis from a 44-week open-label extension study. Pediatr Dermatol. 2020;37(3):490-7. https://doi. org/10.1111/pde.14135. 